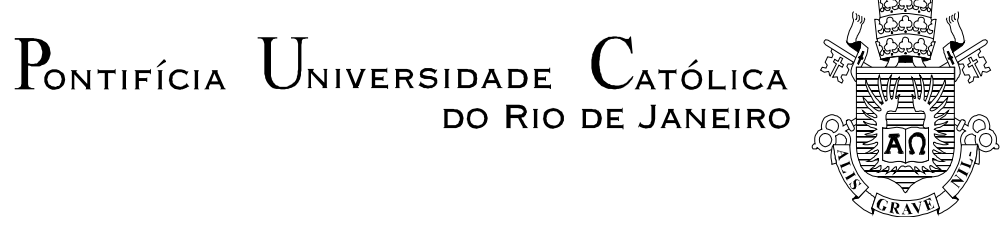

Giovane Barroso Lima Nogueira

Análise do escoamento de emulsões em meios porosos utilizando modelo de rede de capilares

Dissertação de Mestrado

Dissertação apresentada como requisito parcial para obtenção do título de Mestre pelo Programa de PósGraduação em Engenharia Mecânica da PUC-Rio.

Orientador : Prof. Márcio da Silveira Carvalho Co-Orientador: Prof. Vladimir Alvarado 


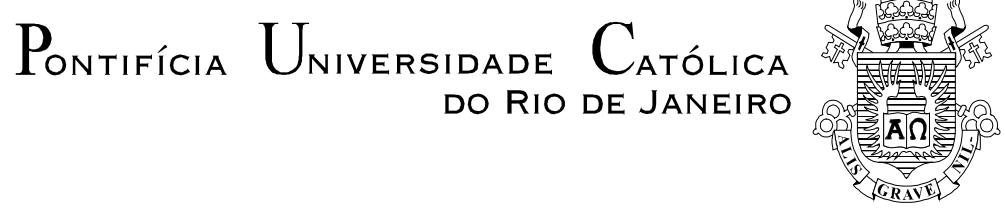

Giovane Barroso Lima Nogueira

\title{
Análise do escoamento de emulsões em meios porosos utilizando modelo de rede de capilares
}

Dissertação apresentada como requisito parcial para obtenção do título de Mestre pelo Programa de Pós-Graduação em Engenharia Mecânica do Departamento de Engenharia Mecânica do Centro Técnico Científico da PUC-Rio. Aprovada pela Comissão Examinadora abaixo assinada.

\author{
Prof. Márcio da Silveira Carvalho \\ Orientador \\ Dep. de Engenharia Mecânica - PUC-Rio
}

Prof. Vladimir Alvarado Co-Orientador

Dep. of Chemical and Petroleum Engineering - Un. of Wyoming

Prof. Angela Ourivio Nieckele

Dep. de Engenharia Mecânica - PUC - Rio

Prof. José Américo de Miranda Neto Dep. de Física - Universidade Federal de Pernambuco

Prof. José Eugenio Leal

Coordenador Setorial do Centro Técnico Científico - PUC-Rio

Rio de Janeiro, 11 de abril de 2011. 
Todos os direitos reservados. É proibida a reprodução total ou parcial do trabalho sem autorização da universidade, do autor e dos orientadores.

\section{Giovane Barroso Lima Nogueira}

Graduou-se em Engenharia Mecânica peloInstituto Politécnico do Rio de Janeiro (IPRJ/UERJ) em 2008, ênfase em Termofluidodinâmica - Modelagem Computacional. Anteriormente, atuou como Técnico em Mecânica na indústriae no ensino profissionalizante em soldagem industrial. Atualmente, cursa doutorado em Engenharia Mecânica na PUC-Rio, na área de injeção de emulsão em meios saturados com óleo.

Ficha Catalográfica

Nogueira, Giovane Barroso Lima

Análise do escoamento de emulsões em meios porosos utilizando modelo de rede de capilares / Giovane Barroso Lima Nogueira ; orientador: Márcio da Silveira Carvalho ; co-orientador: Vladimir Alvarado. - 2011.

64 f. : il.(color.) ; $30 \mathrm{~cm}$

Dissertação (mestrado)-Pontifícia Universidade Católica do Rio de Janeiro, Departamento de Engenharia Mecânica, 2011.

Inclui bibliografia

1. Engenharia mecânica - Teses. 2. Escoamento de emulsão. 3. Meios porosos. 4. Modelo de rede de capilares. 5. Controle da mobilidade. I. Carvalho, Márcio da Silveira. II. Alvarado, Vladimir . III. Pontifícia Universidade Católica do Rio de Janeiro. Departamento de Engenharia Mecânica. IV. Título.

CDD: 621 
Para a minha esposa,

Schaiane Nogueira Ouverney Barroso, por tudo que ela representa na minha vida. 


\section{Agradecimentos}

Gostaria de agradecer imensamente à minha esposa, Schaiane Barroso, por partilhar cada instante intensificado pelas experiências nesses dois anos; desde os momentos do silêncio da dúvida e temor aos episódios de alegria pelos sucessos obtidos. Sua temperança diante às diferenças neste período fizeram aumentar ainda mais minha admiração pela mulher que amo.

À minha família, agradeço pelo incentivo e confiança durante este caminho. Minha mãe, Ledir Barroso, e meu irmão, Joselei Barroso e família, tiveram um papel essencial nestes dois anos.

Ao meu orientador, prof. Márcio Carvalho, pela supervisão durante meu curso de mestrado e em cada etapa desta pesquisa. Seu constante apoio e confiança no meu trabalho fizeram parecer mais tênue este sinuoso caminho. Meu co-orientador, prof. Vladimir Alvarado, com seu profundo conhecimento, completou esta produtiva parceria.

Aos responsáveis pelo Consórcio para Modelagem em Micro-Escala do Imperial College London, pela disponibilização da rede de capilares em seu website.

Dedico este trabalho a todos os citados acima, aos professores do Departamento de Engenharia Mecânica e aos funcionários da secretaria do departamento e das bibliotecas da PUC-Rio. Agradeço a Deus por cada momento vivido até aqui e por todas as pessoas que passaram pela minha vida, aos colegas e em especial a todos os professores que contribuíram à minha formação.

Agradeço à agência de fomento CAPES pelo apoio financeiro e à PUC-Rio pela oportunidade de desenvolvimento desta pesquisa e de obtenção do grau de Mestre em Engenharia Mecânica. 


\section{Resumo}

Nogueira, Giovane Barroso Lima; Carvalho, Márcio da Silveira. Análise do escoamento de emulsões em meios porosos utilizando modelo de rede de capilares. Rio de Janeiro, 2011. 64p. Dissertação de Mestrado - Departamento de Engenharia Mecânica, Pontifícia Universidade Católica do Rio de Janeiro.

Emulsões podem ser utilizadas como agentes de controle de mobilidade em diferentes processos de recuperação melhorada de petróleo e armazenamento de carbono em reservatórios porosos. A aplicação desta técnica, com a escolha correta das características das emulsões injetadas e a determinação das condições de operação ótimas, requer um entendimento adequado do escoamento de emulsões em meios porosos. As características macroscópicas do fluxo de emulsões através de meios porosos estão diretamente ligadas ao escoamento bifásico na escala de poros. Modelos de rede de capilares permitem a implementação dos mecanismos de fluxo das gotas nas gargantas de poros e fornecem parâmetros macroscópicos do escoamento. Neste trabalho, o escoamento de emulsões em meios porosos é analisado através de um modelo dinâmico de rede de capilares tridimensional e não-estruturada. A distribuição de pressão nos poros, e consequentemente o fluxo em cada capilar da rede, é determinada pelo balanço de massa em cada poro. O efeito das gotas da fase dispersa no comportamento do escoamento em cada elemento da rede é descrito por um fator de bloqueio de fluxo baseado em resultados experimentais de escoamento de emulsões através de micro capilares com gargantas. $\mathrm{O}$ fator de bloqueio descreve a mudança da condutividade de cada elemento e é uma função da geometria da garganta, do tamanho e concentração volumétrica da fase dispersa e do número de capilaridade local. A distribuição de concentração da fase dispersa ao longo da rede é descrita através de uma equação de transporte de massa, permitindo assim o estudo do processo de filtragem de gotas nos poros e o estudo da injeção alternada de água e emulsão. A integração no tempo do modelo dinâmico é feita por um método semi-implícito e o sistema de equações não linear obtido a cada passo de tempo é resolvido através de um método iterativo. Os resultados apresentam a evolução da redução da permeabilidade e concentração de gotas na saída do meio poroso em função do tamanho das gotas, da vazão de injeção, da concentração da emulsão injetada e do volume injetado de emulsão. A análise da injeção alternada de água-emulsão mostra claramente o bloqueio de poros por gotas da emulsão e a alteração no padrão de escoamento após reiniciada a injeção de água.

\section{Palavras-chave}

Escoamento de emulsão; meios porosos; modelo de rede de capilares; controle da mobilidade. 


\section{Abstract}

Nogueira, Giovane Barroso Lima; Carvalho, Márcio da Silveira (Advisor).Analysis of emulsion flow through porous media using capillary network model. Rio de Janeiro, 2011. 64p. MSc. Dissertation - Departamento de Engenharia Mecânica, Pontifícia Universidade Católica do Rio de Janeiro.

Emulsions can be used as mobility control agents in different enhanced oil recovery and carbon storage methods in oil reservoirs. The application of this technique, with the correct choice of the injected emulsion characteristics and the determination of optimal operating conditions, requires an adequate understanding of the emulsion flow in porous media. The macroscopic characteristics of the emulsion flow through porous media are directly linked to the two-phase flow at the pore scale. Capillary network models allow the implementation of the drop flow mechanisms in the pore throats and the determination of macroscopic flow parameters. In this work, emulsion flow in porous media is analyzed through an unstructured 3D dynamic network model. The pressure distribution, and consequently the flow rate in each capillary of the network, isdetermined by mass balance equation in each pore. The effects of the drops of dispersed phase in the flow behavior within each element of the network is described by a flow blocking factor based on experimental results on emulsion flow through single microcapillary tubes with throats. The blocking factor describes the changes in the conductivity of each element and it is a function of the throat geometry, the size and volumetric concentration of the dispersed phase and the local capillary number. The concentration distribution of the dispersed phase along the network is described by a mass transport equation, allowing the study of the filtration process of the drops in the pores and the analysis of the alternate injection of water and emulsion. Time integration in the dynamic model is performed by a semi-implicit method and the non-linear system of equations obtained in each time step is solved by an iterative method. The results illustrate the evolution of the permeability reduction and the effluent concentration of drops as a function of the drops size, injection flow rate, concentration of the injected emulsion and injected volume of emulsion. The analysis of the emulsion/water alternate injection clearly shows the pore blockage by the emulsion drops and the change in the flow pattern after the reinjection of water.

\section{Keywords}

Emulsion flow; porous media; capillary network model; mobility control. 


\section{Sumário}

1 Introdução 10

1.1. Descrição do problema 12

1.2. Controle da mobilidade através da injeção de emulsão 13

1.3. Revisão bibliográfica 14

1.4. Objetivos 19

2 Modelo de rede de capilares $\quad 21$

2.1. Topologia da rede 21

2.2. Geometria do espaço poroso 23

2.3. Modelo de escoamento 26

2.3.1. Escoamento em regime permanente 26

2.3.2. Condutância hidráulica 28

2.3.3. Modelo dinâmico do escoamento 31

2.3.4. Passo de tempo 33

3 Comportamento das emulsões em meios porosos 35

3.1. Escoamento de emulsões em tubos capilares 37

3.2. Aplicação do fator de bloqueio na rede de capilares 37

3.3. Fator de bloqueio 39

3.4. Concentração volumétrica de gotas na emulsão 41

3.5. Modelo computacional 43

4 Resultados e conclusões $\quad 48$

4.1. Injeção contínua de emulsão em rede saturada com água 49

4.1.1. Influência da vazão de injeção e tamanho de gotas 49

4.1.2. Influência da concentração de gotas 52

4.2. Injeção alternada de emulsão e água 54

4.2.1. Influência do volume de emulsão injetado 56

5 Considerações finais $\quad 59$

6 Referências bibliográficas 61 


\section{Lista de figuras}

Figura 1.1 Histórico e projeção do consumo mundial de energia e preços do petróleo 10

Figura 1.2 Amostra de rocha-reservatório e estrutura da rede de capilares

Figura 2.1 Arranjo básico de meio poroso e sua representação via modelo de rede

Figura 2.2 Distribuição dos raios inscritos dos poros e capilares da rede utilizada

Figura 2.3 Formatos das seções transversais dos elementos da rede

Figura 2.4 Estrutura elementar de poro e capilar para cálculo das

Figura 3.1 Micrografia de tubo capilar com gotas de emulsão deformadas

Figura 3.2 Modelos dos mecanismos de atuação da emulsão sugeridos:

a) análise reológica, b) retardamento das gotas e c) captura de gotas

Figura 3.3 Fator debloqueioem fluxo de emulsão em tubo capilar

Figura 3.4 Influência do tamanho de gota e número de capilaridade no fator de bloqueio

Figura 3.5 Esquema da injeção de emulsão em a) um elemento saturado com óleo e b) um arranjo de elementos saturados com água

Figura 3.6 Fluxograma referente ao código desenvolvido

Figura 4.1 Curvas típicas de permeabilidade e concentração na saída da rede para injeção contínua de emulsão

Figura 4.2 Redução na permeabilidade e permeabilidade final em função da vazão de injeção para tamanhos de gota fixos

Figura 4.3 Redução na permeabilidade e permeabilidade final em função do tamanho de gota para vazão fixa

Figura 4.4 Curvas típicas de permeabilidade e concentração na saída da rede para injeção de emulsão com diferentes concentrações

Figura 4.5 Resultados das simulações com maior e menor bloqueio obtidas para injeção contínua

Figura 4.6 Redução na permeabilidade e permeabilidade final em função da concentração da emulsão, para vazão e tamanhos de gota fixos

Figura 4.7 Curvas de permeabilidade e concentração da injeção alternada

Figura 4.8 Evolução da permeabilidade durante a injeção alternada com concentração constante

Figura 4.9 Vazão nos capilares de seção transversal da rede (a) com as respectivas intensidades de bloqueio local (b) 